\title{
Recent and future manifestations of a contingent global mean sea level acceleration
}

DOI: https://doi.org/10.1515/jogs-2020-0115

Received August 7, 2020; accepted January 8, 2021

\begin{abstract}
We analyzed globally averaged satellite altimetry mean sea level time series during $1993-2018$ and their future manifestations for the following 25 years using a kinematic model, which consists of a trend, a contingent uniform acceleration, and a random error model. The analysis of variance results shows that the model explains $71.7 \%$ of the total variation in global mean sea level for which $70.6 \%$ is by the secular trend, and $1.07 \%$ is due to a contingent uniform acceleration. The remaining $28.3 \%$ unexplained variation is due to the random errors, which are dominated by a first order autoregressive process driven mostly by oceanic and atmospheric variations over time. These numbers indicate more bumps and jumps for the future manifestations of the global mean sea level anomalies as illustrated using a one-step ahead predictor in this study. Our findings suggest preponderant random errors are poised to further confound and negatively impact the certitude of published estimates of the uniform global sea level acceleration as well as its prediction under an increasingly warmer Earth.
\end{abstract}

Keywords: Satellite altimetry; Global mean sea level trend and acceleration; Global mean sea level prediction; Climate change

Averages play two tricks: first, they put life's lumps and bumps through the blender...

M. Blastland \& A. Dilnot, The Numbers Game, 2009.

\section{Introduction}

Evidence for global mean sea level (GMSL) rising faster during the 20th and $21^{\text {st }}$ centuries resulting from an increasingly warming Earth is an important indicator in

\footnotetext{
*Corresponding Author: H. Bâki İz: Division of Geodetic Science, School of Earth Sciences, The Ohio State University, Columbus, Ohio, USA, E-mail: H.Baki.iz@gmail.com

C.K. Shum: Division of Geodetic Science, School of Earth Sciences, The Ohio State University, Columbus, Ohio, USA
}

assessing anthropogenic contributions to the complex climate change feed-back system. Recently, Nerem, et al. (2018) reported $0.084 \pm 0.025 \mathrm{~mm} / \mathrm{yr}^{2}$ (68\% confidence level, CL) GMSL acceleration since 1993, which is inferred from the globally averaged satellite altimetry (SA) time series. Subsequently, Ablain et al. (2019) furnished another estimate of GMSL acceleration, $0.12 \pm 0.07 \mathrm{~mm} / \mathrm{yr}^{2}(90 \%$ $\mathrm{CL})$. However, the reported estimates for uniform accelerations at globally distributed tide gauge, TG, stations with century long records are preponderantly smaller than the acceleration estimated from globally averaged SA data (Iz and Shum 2019). Kleinherenbrink et al (2019) assessed plausible errors due to altimetry instrument (internal path delays due to thermal variations), and geophysical correction (wind/wave dependent sea state bias), and concluded that their estimated global mean acceleration using altimetry sea-level data have primarily a range of $0.015-$ $0.019 \pm 0.026 \mathrm{~mm} / \mathrm{yr}^{2}$ and therefore statistically not significant at the 95\% confidence level. Moreover, a recent study by Iz and Shum (2020) challenged the SA based GMSL uniform acceleration on the basis of unmodelled systematic confounders. They demonstrated that the non-linear globally averaged sea level anomalies can be explained equally well by a number of previously established periodicities in sea levels (Iz 2014 and 2015), which were not taken into consideration by the recently reported SA based uniform GMSL acceleration estimates.

This study will also operate on a model that includes a uniform (constant) acceleration representation. As briefly discussed above, the presence and uniformity of the GMSL acceleration are not established with certitude. Consequently, the GMSL acceleration will be called $a$ contingent uniform GMSL acceleration as a reminder of this important distinction throughout the narrative.

In this study, we expose another pitfall in detecting global uniform sea level acceleration during SA era, and its predictions by demonstrating the manifestations of the unmodeled global sea level anomalies caused by the random effects intensified by a first-order autoregressive AR(1) process. The AR(1) correlation coefficient of the GMSL SA time series is estimated to be $\hat{\rho}=0.935$, which reduces the effective sample size of the monthly GMSL time series 
from 25 years down to 10 months! Such a drastic impact of the unexplained random disturbances on solution statistics necessitates an in-depth analysis of their effects on the magnitudes of the current and future manifestations of the GMSL anomalies.

In the following sections, we first gave a brief description of the globally averaged SA monthly time series to be used in this study. We then used a kinematic model with a secular trend and a contingent uniform acceleration to represent GMSL variations as observed by SA. A detailed Generalized Least Squares, GLS, solution was reported together with the results of an analysis of variance, ANOVA, study. We then summarized the two approaches in predicting future GMSL anomalies due to a contingent uniform global sea level acceleration and illustrated sampled manifestations of future GMSL anomalies for a visual as well as quantitative assessment of the predicted variations for the recent the contingent GMSL acceleration.

\section{Global Mean Sea Level Data}

This study utilizes monthly averaged GMSL data, shown in Figure 1, which were generated using the Integrated Multi-Mission Ocean Altimeter Data for Climate Research (GSFC, 2017). It combines Sea Surface Heights from TOPEX/Poseidon, Jason-1 and OSTM/Jason-2 with all systematic errors corrected and placed onto a georeferenced orbit (Beckley, et al., 2016). Unfortunately, no uncertainties for the month by month averaged anomalies were available.

A critical issue for the SA data sets, as mentioned before, was raised by Kleinherenbrink et al. (2019). Their assessment of the globally averaged SA data uncertainties rendered the GMSL acceleration claimed by Nerem at al., 2018, and Ablain et al., 2019) statistically not significant at a 95\% confidence level. Nonetheless, our inquiries to obtain the revised data used in their study to replicate their result were unanswered. Hence, we will assume that the above data set is still viable for this investigation because this series was also nearly replicated by the other data centers (vox populi).

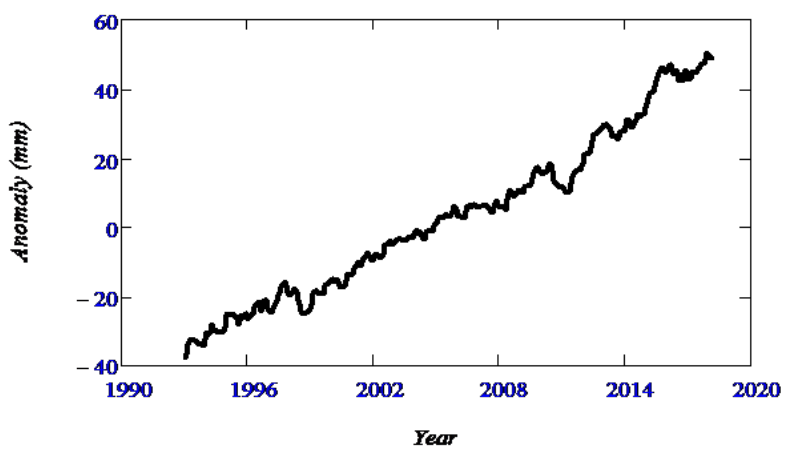

Fig. 1. Globally averaged mean mean sea level anomaly as observed by SA during 1993 - 2018 (GSFC, 2017).

\section{A kinematic model with a trend and a contingent uniform GMSL acceleration}

The following kinematic model will be under consideration to represent the GMSL anomalies with a secular trend $v$ and a contingent uniform acceleration $a$,

$$
h_{t}=h_{t_{0}}+v\left(t-t_{0}\right)+\frac{1}{2} a\left(t-t_{0}\right)^{2}+e_{t}
$$

In this model the time index is denoted by $t=1 \ldots T$, where $T$ is the number of months and includes all the available globally averaged SA data denoted by $h_{t}$. The intercept $h_{t_{0}}$ is the reference height of the GMSL time series defined at the reference epoch $t_{0}$ chosen to be in the middle of the series. The intercept $h_{t_{0}}$ is to be estimated together with the parameters for the global velocity $v$ and the contingent acceleration $a$.

The statistical properties of the model are as follows. The disturbances, , are first-order autocorrelated, i.e.

$$
e_{t}=\rho e_{t-1}+u_{t} \quad 0 \leq|\rho|<1
$$

where $\rho$ is the correlation coefficient of the AR(1). The random noise is $u_{t} \sim\left(0, \sigma_{u}^{2}\right)$, and $\sigma_{u}^{2}$ is the variance of the random error (shocks/innovations). Disturbances $e_{t}$ and random noise $u_{t}$ are related to each other as follows (Toutenburg, 1982, pg. 24),

$$
E\left(e_{t}\right)=0, \quad \operatorname{var}\left(e_{t}\right)=\sigma^{2}=\sigma_{u}^{2}\left(1-\rho^{2}\right)^{-1}
$$

In this formulation, we model the $T \times T$ variance covariance (V/C) matrix of the first-order autocorrelated disturbances $\Sigma$ as,

$$
\Sigma=\sigma^{2} \cdot\left[\begin{array}{ccccc}
1 & \rho & \rho^{2} & \cdots & \rho^{T-1} \\
\rho & 1 & \cdots & \cdots & \rho^{T-2} \\
\vdots & \vdots & \vdots & \ddots & \vdots \\
\rho^{T-1} & \rho^{T-2} & \rho^{T-3} & \cdots & 1
\end{array}\right]
$$


The autocorrelation decreases for increasing time lag because $|\rho|<1$. The correlation between two random variables $e_{t}$ and $e_{t-\tau}$ is $\sigma^{2} \rho^{\tau}$, where $\tau$ is the time lag. The above patterned $\mathrm{V} / \mathrm{C}$ matrix has an analytical inverse and is given by,

$$
\begin{aligned}
& \Sigma^{-1}=\frac{\sigma^{-2}}{1-\rho^{2}} \mathbf{W} \\
& =\left[\begin{array}{cccccc}
1 & -\rho & 0 & \cdots & 0 & 0 \\
-\rho & 1+\rho^{2} & -\rho & \cdots & 0 & 0 \\
0 & -\rho & 1+\rho^{2} & \cdots & 0 & 0 \\
\vdots & \vdots & \vdots & \ddots & \vdots & \vdots \\
0 & 0 & 0 & \cdots & 1+\rho^{2} & -\rho \\
0 & 0 & 0 & \cdots & -\rho & 1
\end{array}\right]
\end{aligned}
$$

where $\mathbf{W}$ is a $T \times T$ symmetric positive definite matrix (ibid). The unknown autocorrelation coefficient that appears in the above expression can be estimated by carrying out GLS solutions for various autocorrelation coefficients within the interval $0 \leq|\rho|<1$. The solution that minimizes the standard error of the solution $\sigma$ and/or DurbinWatson statistic, DW, that is closer to the its expected value 2 gives the optimal correlation between adjacent disturbance terms (Neter et al., 1996).

\section{Solution}

We estimated the model parameters described in the previous section using a GLS solution. The trend and the contingent uniform acceleration estimates are $3.27 \pm 0.10 \mathrm{~mm} / \mathrm{yr}$., and $0.081 \pm 0.030 \mathrm{~mm} / \mathrm{yr}^{2}$, respectively. The variance inflation factor, VIF ${ }^{1}$, between the two parameters is close to 1 , which permits us to focus only on the acceleration without interfering the trend estimate in the following sections.

The classical ANOVA computations carried out together with the GLS solution reveal that the model explains $71.7 \%$ of the total variation in the GMSL for which $70.6 \%$ belongs to the trend, $1.07 \%$ is due to the contingent uniform acceleration and the remainder $28.3 \%$ are the are the autocorrelated random effects driven by episodic random shocks/innovations as they will be elucidated later. Among the other statistics, the estimated AR(1) correlation coefficient is $\hat{\rho}=0.935$. This estimate is unexpectedly large as compared to the AR(1) experienced at glob-

1 VIF is a measure of the linear dependence among the columns (coefficients of the parameters) of the coefficient matrix interpreted in the context of its impact in inflating the variances of the estimates. A VIF factor close to 1 is an indicator of the independence of a variable from the linear combination of the other variables. ally distributed TG stations, which remain mostly within the interval [0.1 - 0.4] (Iz, 2014). As we stated before, the $\mathrm{AR}(1)$ process is important in assessing the reliability of the recent GMSL trend and acceleration and in predicting the future GMSL anomalies especially when such correlations are large.

Inserting the estimated model parameters for the trend and the contingent acceleration into eqn. (1) enables us to compute the residuals $\hat{e}_{t}$. The estimated correlation coefficient for AR(1) in eqn. (2) allows us also estimating the underlying random noise. Both times series are displayed in Figure 2.

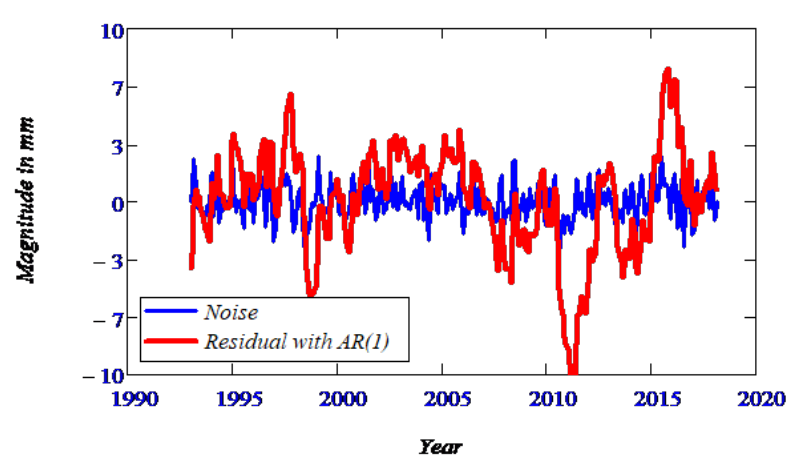

Fig. 2. Residual anomalies and the underlying random noise.

The estimated standard error of the disturbances $\hat{\sigma}$ calculated using the following relationship is about $2.89 \mathrm{~mm}$ for the $T \times 1$ vector of autoregressive residual vector, $\hat{\mathbf{e}}$,

$$
\hat{\sigma}=\sqrt{\frac{\hat{\mathbf{e}}^{T} \mathbf{W}^{-1} \hat{\mathbf{e}}}{T-3}}=2.89 \mathrm{~mm}
$$

from which $\hat{\sigma}_{u}$ was calculated using,

$$
\hat{\sigma}^{2}=\hat{\sigma}_{u}^{2}\left(1-\hat{\rho}^{2}\right)^{-1} \rightarrow \hat{\sigma}_{u}=1.06 \mathrm{~mm}
$$

Eqn. (2) was also used to calculate the random noise from which their standard deviation was determined to be 1.05 $\mathrm{mm}$.

Although the basis of the noise is random, its impact on the unmodeled GMSL variability, i.e. residuals is almost three times larger because of the AR(1) process. The autoregression causes bumps and jumps in the residuals during their time evolution as shown in Figure 2.

Plotting residuals against the adjusted GMSL anomalies $^{2}$ is intended to explore systematic unmodelled effects visually. Figure 3 shows that residuals are not systematic

2 Adjusted GMSL anomalies are obtained by adding the residuals to the observed GMSL anomalies. 
but exhibit transient abrupt changes induced by the random noise, termed as shocks or innovations in macroeconomics (Qin and Gilbert, 2001). These residual anomalies are important to understand the recent claims about the uniform global sea level acceleration (Nerem et al., 2018 and Ablain et al., 2019). Both studies deployed an ordinary least squares, OLS, solution in their estimation of trend and uniform acceleration ignoring the $\mathrm{AR}(1)$ process then corrected the uncertainties of the estimates for the AR(1) effects, or for the other source of error in the study by Ablain et al. (2019). The use of OLS may seem to be viable theoretically because the expected values of the OLS estimates are the same as contrasted to the one obtained using GLS solution, yet it may potentially be misleading under certain conditions, which we will examine in the subsequent sections.

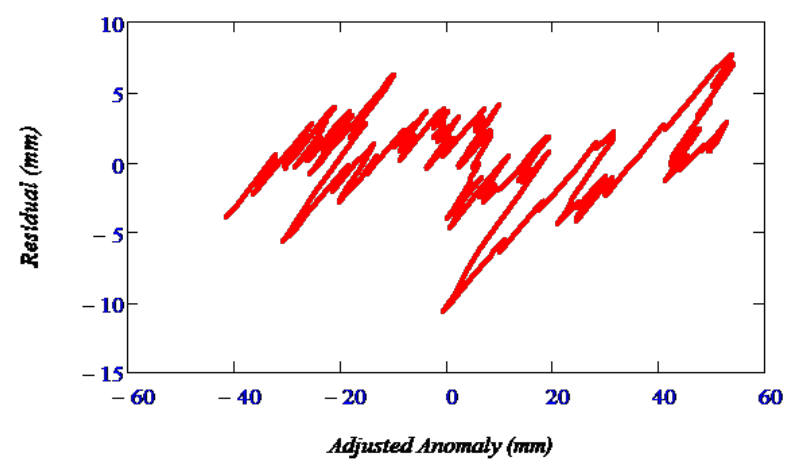

Fig. 3. AR(1) residuals are plotted against the adjusted anomalies. They reveal unpredictable bursts, caused by random schocks/innovations, over time.

The lagged autocorrelations of the residual and the random noise shown in the correlogram (Figure 2) confirm the random nature of the noise and $\mathrm{AR}(1)$ properties of the residuals. The AR(1) is maximum for monthly intervals. The magnitudes of the subsequent autocorrelations diminish rapidly until the 8 month lags, beyond which there are no statistically significant lagged effect at $95 \%$ CL.

The outcome of the GLS solution can be summarized visually in Figure 5 and Figure 6. Figure 5 displays the prepondorance of the trend in observed GMSL anomalies, their adjusted values inclusive of acceleration, and the smooth predicted $^{3}$ variability. In this presentation, nonlinearity of the uniform acceleration is barely perceptible in

3 Prediction does not always refer to a future behavior but also to the present by referring to the adjusted observations calculated using estimated model parameters.

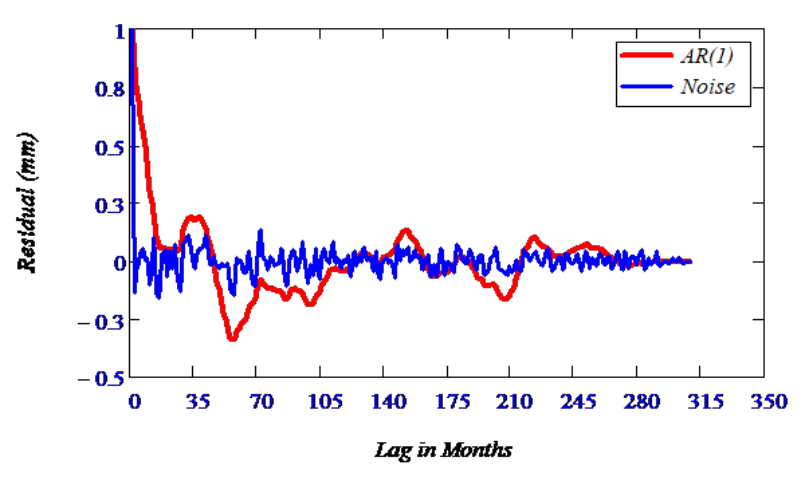

Fig. 4. Correlogram for AR(1) residuals and noise.

comparison to the observed random variability. Figure 6 provides visual clarity to assess how important the first order autocorrelation is in relation to the acceleration signal and the underlying random noise as we emphasized in the introduction section. This unmodeled variability is critical since it will be carried over the future manifestations of the global sea level variations and must be accounted for in predictions.

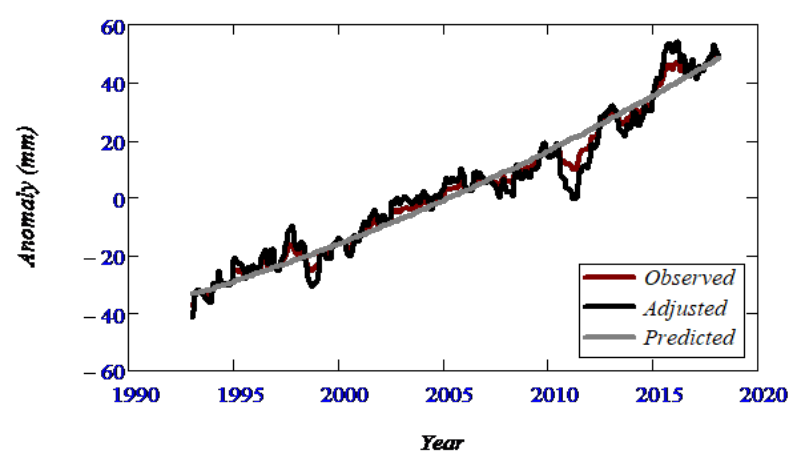

Fig. 5. The visual synopsis of the GLS solution inclusive of the trend and the contingent acceleration during 1993 - 2018.

\section{The origin of the autoregressive disturbances}

Although we have demonstrated the AR(1) properties of the disturbances, the random nature of the underlying noise cannot be well established. The noise exhibits randomness (Figure 2 and Figure 6), free from systematic variations visually, but they do not pass the runs test for randomness at 95\% significance level. Yet, as discussed in Iz and Shum (2020) and Nerem et al. (2018), some of the unexplained GMSL anomalies during the SA period can be at- 


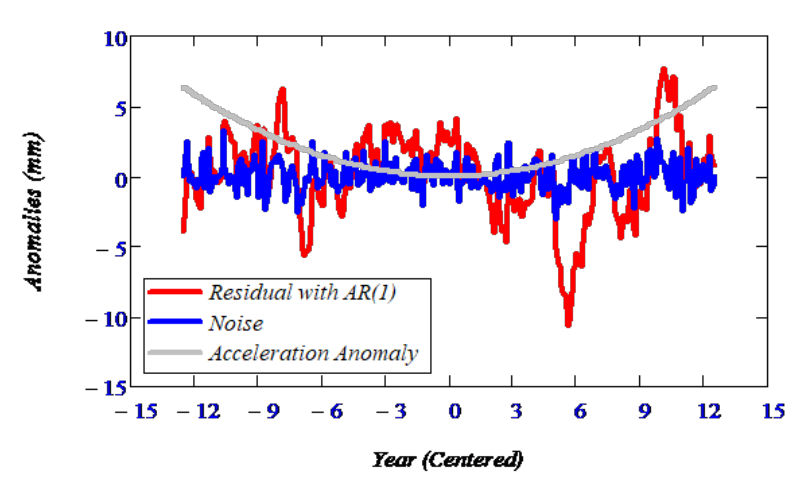

Fig. 6. The visual synopsis of the GMLS anomalies, centered at the middle epoch of the series, due by the estimated contingent and uniform acceleration only.

tributed Multivariate ENSO Index , MEI, which combines both oceanic and atmospheric variables, and facilitates assessing the effect of ENSO in a single index. The index gives real-time indications of ENSO intensity through historical analysis of various data (NOAA, 2019). The residuals and MEI time series displayed in Figure 7 have a maximum cross-correlation 0.5 , which occurs with a time lag of 4 months. This finding suggests that SA measurement and its data processing errors are not solely responsible for the autoregressive behavior of the residuals. More importantly, the cross correlation between the residuals and MEI evokes the necessity of using residuals in GMSL predictions because they are integral the part of a series of naturally occurring phenomena. This is the topic of the following sections.

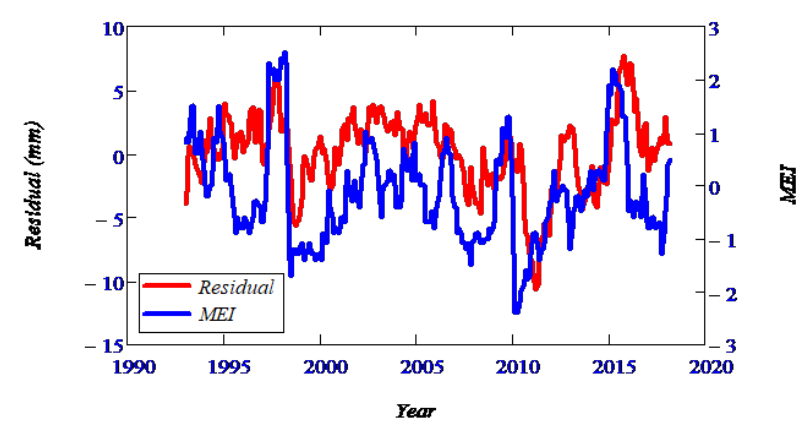

Fig. 7. $M E I$ and the $G L S$ residuals $(\mathrm{mm})$ during the SA period.

\section{Prediction of GMSL anomalies}

Recent publication by Nerem et al (2018) projected their uniform GMSL acceleration findings till 2100. Such pro- jections are safe but of limited use, especially at local scales. In particular, the ways sea level projections were conducted in lieu of predictions are too simplistic for such an important topic (see Bray D., and H. von Storch, 2009 for an in-depth comparison between projections and predictions). Because SA is capable of observing as well as predicting GMSL variations with precision, it is the pillar of the climate change science by establishing baselines against which all climate related projections can be tested and verified. Here, we introduce two different approaches to initiate a discussion as well as to gain insight about the manifestations of GMSL anomalies not only for the future but also for shedding light into the present.

Classical predictions based mean square error, MSE, criterion provide information about the expected values of future events. They are pure and smooth in theory, but lose precision in the real world, as if they are two different realities. Predictions built on expected values of the model parameters, neatly defined around the edges, become an exercise in suppressing realities providing a false clarity. Predictions based on MSE criterion serve well for now for assessing the GMSL budget when precision is not a mandate, yet the lesser known predictions based on the MSE of Prediction, MSEP, are rough on the edges and more informative as demonstrated by Iz, et al. (2012), and Iz (2018), because they account for the effect of random variations that dissipates in classical predictions and indispensable for coastal studies. In the following section, we provide summaries of the operational formularies based on the publications by Goldberger (1962), Tautenburg (1977), and Toutenburg (1982). The MSEP criterion was first introduced to the Geodetic literature by Schaffrin (1983), and Iz (1987) examined its applications for the analyses of crustal deformations.

\section{Least Squares Prediction Based on MSE}

Consider the following linear model,

$$
\mathbf{y}=\mathbf{X b}+\mathbf{e}, \quad \mathbf{e} \sim\left(\mathbf{0}, \sigma^{2} \mathbf{W}\right)
$$

where,

$\mathbf{y}$ the $\mathrm{T} \times \mathrm{x}$ vector of the dependent variable, is random and observable

$\mathbf{X}$ the T $\mathrm{x}$ K matrix of explanatory variables, is known and fixed

b the $\mathrm{K} \times 1$ vector of regression coefficients, is fixed and unobservable

e the $\mathrm{T} \times 1$ vector of disturbances, is random and unob- 
servable

$\Sigma=\sigma^{2} \mathbf{W}$ the T $\mathrm{x}$ T dispersion matrix is unobservable.

$\sigma^{2}$ the variance of the disturbances.

The prediction equation that follows is,

$$
\mathbf{y}_{\star}=\mathbf{X}_{\star} \mathbf{b}+\mathbf{e}_{\star} \mathbf{e}_{\star} \sim\left(\mathbf{0}, \sigma^{2} \mathbf{W}_{\star}\right)
$$

Where $\mathbf{y}_{\star}$ is the $T \star \times 1$ vector of prediction horizon from which all the other dimensions of the prediction equation and the corresponding statistics can be inferred. In this prediction model, the $\mathrm{V} / \mathrm{C}$ matrix of the future disturbances $\mathbf{e}_{*}$, and their dependence to the recent disturbances $\mathbf{e}$ are given by,

$$
E\left(\mathbf{e}_{\star} \mathbf{e}_{\star}^{T}\right)=\sigma^{2} \mathbf{W}_{\star}, \quad E\left(\mathbf{e} \mathbf{e}_{\star}^{T}\right)=\sigma^{2} \mathbf{W}_{0}
$$

The solution to this classical prediction formulation is based on the following MSE matrix of the predicted $\hat{\mathbf{y}}_{*}$, which is given by,

$$
\operatorname{MSE}\left(\hat{\mathbf{y}}_{\star}\right):=\sum_{\hat{\mathbf{y}}_{\star}}=E\left(\hat{\mathbf{y}}_{\star}-E\left(\mathbf{y}_{\star}\right)\right)\left(\hat{\mathbf{y}}_{\star}-E\left(\mathbf{y}_{\star}\right)\right)^{T}
$$

which predicts,

$$
\hat{\mathbf{y}}_{\star}=\mathbf{X} \star \hat{\mathbf{b}}
$$

where $\hat{\mathbf{b}}$ is the estimated vector of model parameters. The $\mathrm{V} / \mathrm{C}$ matrix $\Sigma_{\hat{\mathbf{y}} \star}$ of predicted observation vector $\hat{\mathbf{y}} \star$ is,

$$
\operatorname{MSE}\left(\hat{\mathbf{y}}_{\star}\right)=\Sigma_{\hat{\mathbf{y}}_{\star}}=\sigma^{2} \mathbf{X}_{\star}\left(\mathbf{X}^{T} \mathbf{W}^{-1} \mathbf{X}\right)^{-1} \mathbf{X}_{\star}^{T}
$$

The resulting predictor is unbiased. In this approach, the interdependence between the random disturbances and future disturbances disappears thanks to MSE target function that is based on the expected values for the future observations. The predicted values are theoretically averaged realizations of their future expected values and consequently, blind to the underlying present and future random variations. A realistic representation of the predicted values is given in the following section.

\section{Least squares prediction based on MSEP}

Considering the same linear model given by eqn.(8), an alternative predictor is obtained by minimizing the following target function MSEP,

$$
\operatorname{MSEP}\left(\hat{\mathbf{y}}_{\star}\right):=E\left(\hat{\mathbf{y}}_{\star}-\mathbf{y}_{\star}\right)\left(\hat{\mathbf{y}}_{\star}-\mathbf{y}_{\star}\right)^{T} .
$$

The resulting predictor is an alternative to the classical least squares predictor. The formulation is based on minimizing the expected value of quadratic distance between the predicted and the true values instead of their expected values of predictions. The predictor $\hat{\mathbf{y}}_{\star}^{4}$ based on MSEP is also an unbiased predictor of $\mathbf{y}_{\star}$ and is given by,

$$
\hat{\mathbf{y}}_{\star}=\mathbf{X} \star \hat{\mathbf{b}}+\mathbf{W}_{\mathbf{0}}^{\mathbf{T}} \mathbf{W}^{-1} \hat{\mathbf{e}}_{\star}
$$

In this expression, the first element of the above relationship is the expectation of the $\mathbf{y} *$, which is the classical least squares predictor given by eqn. (12). The second element is a predictor of the future disturbance vector $\mathbf{e}_{\star}$, which is missing in the classical case. The corresponding MSEP is given by,

$$
\begin{gathered}
\operatorname{MSEP}\left(\hat{\mathbf{y}}_{\star}\right)=\sigma^{2} \mathbf{X}_{\star}\left(\mathbf{X}^{T} \mathbf{W}^{-1} \mathbf{X}\right)^{-1} \mathbf{X}_{\star}^{T}+\sigma^{2} \mathbf{W}_{\star-} \\
-\sigma^{2} \mathbf{X}_{\star}\left(\mathbf{X}^{T} \mathbf{W}^{-1} \mathbf{X}\right)^{-1} \mathbf{X}^{T} \mathbf{W}^{-1} \mathbf{W}_{0^{-}} \\
-\sigma^{2} \mathbf{W}_{0}^{T-1} \mathbf{W}^{-1}\left(\mathbf{X}^{T} \mathbf{W}^{-1} \mathbf{X}\right)^{-1} \mathbf{X}_{\star}^{T}
\end{gathered}
$$

The V/C matrix, $E\left(\mathbf{e}_{\star} \mathbf{e}_{\star}^{T}\right)=\sigma^{2} \mathbf{W}_{\star}$, of the future disturbances $\mathbf{e}_{\star}$ and the interdependence between the future disturbances with the recent disturbances e, i.e., $E\left(\mathbf{e}_{\star}^{T}\right)=$ $\sigma^{2} \mathbf{W}_{0}$, appears in this predictor and in its MSEP. If $\mathbf{W}_{0}=0$ then,

$$
\operatorname{MSEP}\left(\hat{\mathbf{y}}_{\star}\right)=\sigma^{2} \mathbf{X}_{\star}\left(\mathbf{X}^{T} \mathbf{W}^{-1} \mathbf{X}\right)^{-1} \mathbf{X}_{\star}^{T}+\sigma^{2} \mathbf{W}_{\star}
$$

Hence, $\operatorname{MSEP}\left(\hat{\mathbf{y}}_{\star}\right)-\operatorname{MSE}\left(\hat{\mathbf{y}}_{\star}\right)>0$, i.e. positive semi definite, which means that CIs to be established by the MSEP are larger than those given by those from the MSE criterion.

If these a priori information between the past and the future are not readily available, the following tweak (Iz, 2008) still enables predictions as demonstrated in the following section.

\section{One-step-ahead prediction and the future manifestations of the GMSL anomalies due to a contingent acceleration}

A special case of the above predictor is the one-step-ahead predictor and given by,

$$
\hat{\mathbf{y}}_{\star}=\hat{y}_{t_{t^{*}+1}}=\hat{\mathbf{x}}_{t^{t+1}}^{T} \hat{\mathbf{b}}+\mathbf{e}_{\star_{t^{*}+1}} \quad \mathbf{e}_{t_{t^{*}+1}} \sim\left(\mathbf{0}, \sigma_{\star}^{\mathbf{2}}\right)
$$

Considering eqns. (1) and (2), the above expression reduces to the following quantifiable form for predicting future anomalies due to a contingent GMSL acceleration one

4 We did not use a different notation for the predicted values based on MSEP criterion for simplicity. The common notation does not create ambiguity since they are used separately in their own context. 
step at a time,

$$
\hat{y}_{t_{\star}+1}=\frac{1}{2} \hat{a}\left(t_{\star}-t_{\star_{0}}\right)^{2}+\hat{\rho} \hat{e}_{\star t_{\star}}
$$

where $\hat{a}, \hat{\rho}$ and $\hat{\sigma}_{u}^{2}$ are the parameters that were previously estimated using the recent GMSL time series. The expected value of the predictor in the above expression the one-step-ahead predictor based on MSE criterion given by eqn. (12).

We can now generate residuals one-step at a time using a random error based on a normal distribution with mean zero and variance, i.e., $\hat{u}_{t *}\left(0, \hat{\sigma}_{u}^{2}\right)$ together with for a prediction period of equivalent to the current SA time span of $t=1 \ldots T_{\star}$. Having already estimated parameters for a contingent uniform acceleration, future residuals enable us predicting future GMSL anomalies using eqn. (18). A sample of future manifestations are displayed in Figure 8.

The first column of the Figure 8 contrasts the residuals with AR(1) properties and the random errors, namely shocks/innovations that generate them. The second column plots compare them to the predicted anomalies based solely on the contingent acceleration calculated using the classical predictor for a predicted time series centered at the middle epoch (the smooth curve). The plots in the third column contrasts predictions are based on MSE and MSEP together with the root cause of the future variability due to the random shocks/innovations. Because the recent random variations are demonstrably caused by the oceanic and atmospheric variables interactions over time, the sampled future manifestations of the GMSL anomalies in this figure are realistic. They are far from being smooth as their counterparts, i.e. averaged predicted values, which deceptively conceals.

The randomly laden but smoothed averaged anomalies may not be a problem in detecting a GMSL acceleration in the future once the low frequency confounders are identified with increasing time span of the series and the effect of the autocorrelation is properly modeled as demonstrated in this study. But for the present, they may be misleading in detecting and estimating a uniform acceleration, if it exists, during the recent SA period. This is the topic of the next section.

\section{Predicting the present}

As we stated in the footnote earlier, the term prediction also applies to recent events. A GMSL anomaly calculated using the kinematic model with its estimated parameters in place at an epoch during the same period from which they were generated, can also be termed a prediction. In line with this view, we can also treat the future manifestations of GMSL anomalies, as if they may have been alternative realizations of the recent GMSL anomalies during the same period. To investigate the tale-telling of these alternative series for the present, we simulated GMSL anomalies during the SA altimetry period using the estimated sea level acceleration, $0.081 \pm 0.030 \mathrm{~mm} / \mathrm{yr}^{2}$, the AR(1) process with and a normal distribution generator with $u \sim\left(0, \hat{\sigma}_{u}^{2}\right)$, where $\hat{\sigma}_{u}=1.06 \mathrm{~mm}$. We then used these series to estimate again the underlying known contingent acceleration using the GLS as if they were realized during the same SA period (1993 - 2018). We repeated these steps to generate one hundred time series. We found that a large number of the estimated contingent uniform accelerations are concentrated around the underlying acceleration $0.081 \pm 0.030$ $\mathrm{mm} / \mathrm{yr}^{2}$, as expected (Figure 9). Yet, the range of estimates varied within the interval $[0.036-0.126] \mathrm{mm} / \mathrm{yr}^{2}$ suggesting that the current acceleration estimate might have been one of them as well given the marked contribution of the random noise. We also observed that the range of the acceleration estimates diminishes in proportion to the magnitude of the AR(1) correlation coefficient $\hat{\rho}$ used in generating the time series, eqn. (19). In other words, the contingent acceleration estimate depends on the strength of the AR(1) effect, underlying shocks/innovations. The timing of these unmodeled random variations is also important because effects close to the beginning or to the end of the series, are known to have high leverages in estimating model parameters as demonstrated in Iz and Shum (2000). As a result, a shock disseminated through the AR(1) process in the actual GMSL times series may have unduly biased the acceleration estimate. Therefore, given the $1.07 \%$ of the total variability of the contingent uniform acceleration compared to the preponderant $28.3 \%$ unexplained variation due to the random effects, the certitude of the acceleration estimate, despite its statistical significance, is questionable in addition to the other systematic confounders discussed in Iz and Shum (2020).

In this study, we did not quantify the standard error of the predicted anomalies, which can be obtained using eqn. (13) and eqn. (16) simply because, in all likelihood, they will not be a realistic representation of the prediction errors because the globally averaged time series were already preprocessed by the several data centers in order to remove yearly variations for which we have no idea about their magnitudes and their statistical properties. In addition, monthly SA series may have already been overly smoothed using a low pass filter or moving averages, a practice causing loss of information about current and up- 

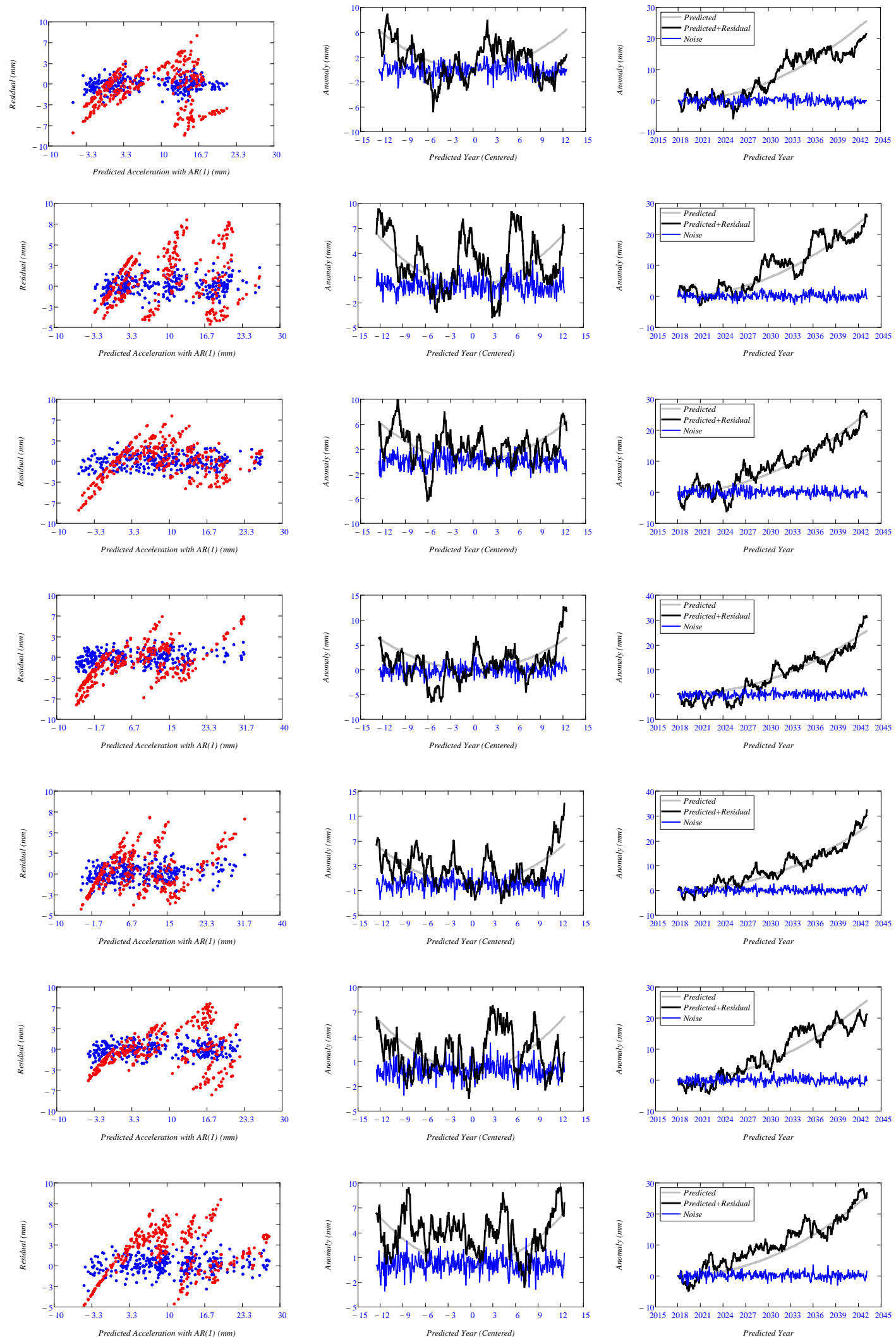

Fig. 8. Future manifestations of the GMSL anomalies due to a contingent acceleration. Contribution due to the trend is not included. 
coming sea level variations realistically as demonstrated in this study.

Another harmful effect of smoothing SA series is the increase in autocorrelation in the globally averaged series. Our previous experience with SA time series at local and regional scale revealed that $\mathrm{AR}(1)$ effects are not large $[0.1-0.4]$ and in step with those experienced at nearby TG station records (Iz et al., 2020). Therefore, part of the markedly large correlation coefficient of the globally averaged SA measurements could be an artifact caused by temporal smoothing and spatial averaging.

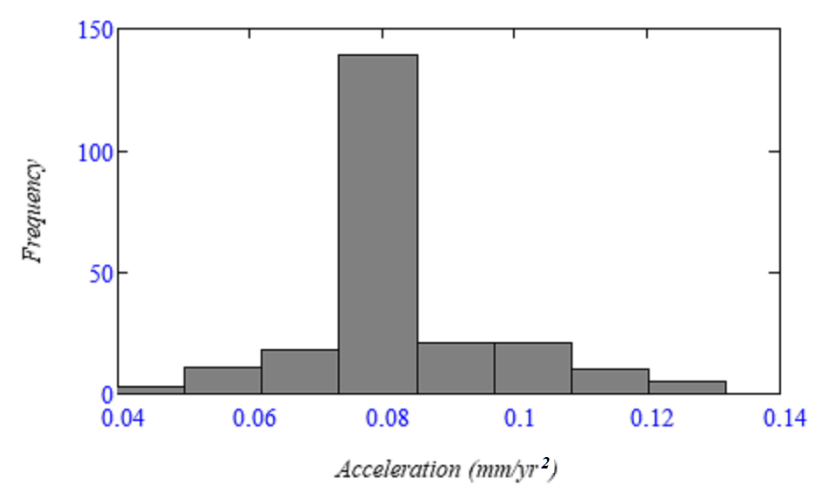

Fig. 9. Distribution of the alternative estimates for the contingent acceleration from the randomly manifested GMSL series for the current period of SA measurements.

\section{Conclusion}

In the past, less than a handful of studies recognized autocorrelations exhibited by sea level time series as observed at TG stations. In the case of globally averaged SA time series, this effect turned out to be strikingly large $(\hat{\rho}=0.935)$ and that it impacts not only investigating the presence of a potential GMSL acceleration but also making predictions about the upcoming GMSL anomalies. In this study, we expanded our earlier analysis of the GMSL variations during 1993 - 2018 using a kinematic model (Iz and Shum 2020) to include an ANOVA study. The ANOVA results revealed that because only $1.07 \%$ of the modeled contingent uniform acceleration explains $71.7 \%$ of the total variation, the AR(1) effect has a marked propensity to bias solution statistics despite the post estimation corrections. Therefore, AR(1) effect has the potential to confound detecting a uniform GMSL acceleration as well as predicting future GMSL anomalies. We also predicted probable future manifestations of GMSL changes due to a contingent acceleration for the following 25 years using a one-step ahead predictor as an alternative to the classical least squares predictor. The formulation based on the alternative predictor accounts for the effect of unmodeled random anomalies in GMSL time series effectively for a realistic representation of the upcoming GMSL anomalies, given the similitude of the residuals and MEI. Our abundantly illustrated predictions manifest more bumps and jumps in GMSL anomalies caused by oceanic and atmospheric effects and their interactions during the upcoming 25 years.

Acknowledgments: We would like to extend our appreciation to the three anonymous reviewers for their time and critical comments.

\section{References}

Ablain M., Meyssignac B., Zawadzki L., Jugier R., Ribes A., Spada G., Benveniste J., Cazenave A., and Picot N., 2019, Uncertainty in satellite estimate of global mean sea level changes, trend and acceleration, Earth Syst. Sci. Data, 11, 1189-1202.

Bibby J. and H. Tautenburg, 1977, Prediction and Improved Estimation in Linear Models, John Wiley and Sons, pgs. 188.

Bray D., and H. von Storch, (2009), "Prediction" or "Projection"? The Nomenclature of Climate Science - Science Communication Vol. 30(4), pgs. 534-543.

Beckley, B., Zelensky, N.P. Holmes, S.A., Lemoine, F.G., Ray, R.D., Mitchum, G.T., Desai, S., Brown, S.T., 2016: Global Mean Sea Level Trend from Integrated Multi-Mission Ocean Altimeters TOPEX/Poseidon Jason-1 and OSTM/Jason-2 Version 4.2. Ver. 4.2. PO.DAAC, CA, USA. Dataset accessed [2018-09-02] at http://dx.doi.org/10.5067/GMSLM-TJ142.

Schaffrin, B., (1983), Model Choice and Adjustment Techniques in the Presence of Prior Information, NASA, Grant No. NSG 5265, OSURF Proj. No. 711055, 37 pp.

Goldberger A. S., 1962, Best Linear Unbiased Prediction in the Generalized Linear Regression Model, Journal of the American Statistical Association, Vol. 57:298, 369-375

GSFC., 2017, Global Mean Sea Level Trend from Integrated Multi-Mission Ocean Altimeters TOPEX/Poseidon, Jason-1, OSTM/Jason-2 Version 4.2 Ver. 4.2 PO.DAAC, CA, USA. Dataset accessed [2018-09-01] at http://dx.doi.org/10.5067/GMSLMTJ42.

Iz, H.B., C. K. Shum, T. Y. Yang, 2020: Conflation of satellite altimetry and tide gauge records at coast, Preprint, DOI: 10.13140/RG.2.2.16423.29603.

İz H.B., C.K. Shum, C.Y. Kuo, 2018, Sea Level Accelerations at Globally Distributed Tide Gauge Stations During the Satellite Altimetry Era, J. Geod. Sci. Vol. 8, pp. 130-135.

İ H.B., 2018, Why and How to Predict Sea Level Changes at a Tide Gauge Station with Prediction Intervals, J. Geod. Sci. Vol. 8, pp. 121-129.

İz H.B., 2015, More confounders at global and decadal scales in detecting recent sea level accelerations, J. of Geod. Sci, 5, 192198. 
İz H.B., 2014, Sub and super harmonics of the lunar nodal tides and the solar radiative forcing in global sea level changes, J. of Geod. Sci., 4, 150-165.

İz H.B. and C.K. Shum, 2000, Mean Sea Level Variation in the South China Sea from Four Decades of Tidal Records in Hong Kong, Marine Geodesy, Vol. 23, No. 4, pp. 221-233.

Iz, H.B., 1987, An Algorithmic Approach to Crustal Deformation Analysis, PhD dissertation, NASA NGS 5265, OSURF 711055, 129 pp.

Kleinherenbrink M., Riva R., Scharro R., 2019, A revised acceleration rate from the altimetry-derived global mean sea level record, Nature, Scientific Reports, Vol. 9.

Nerem R. S., B. D. Beckley, J. T. Fasullo, B. D. Hamlington, D. Masters and G. T. Mitchum, 2018, Climate-change-driven accelerated sea-level rise detected in the altimeter era. PNAS, 1-4.

Neter J.M., Kutner H., Nachtsheim C. J, and Wasserman M., 1996, Applied linear statistical models, Richard D. Irwin, 1408.

Qin, D., and Gilbert C.L., 2001, The Error Term in the History of Time Series Econometrics. Econometric Theory, vol. 17, no. 2, pp. 424-450.

Toutenburg, H., 1982, Prior information in linear models, John Wiley and Sons, New York.

\section{Appendix: List of abbreviations}

\begin{tabular}{llll}
\hline ANOVA & Analysis of Variance & MSE & Mean Square Error \\
AR(1) & Autocorrelation of First Order & MSEP & Mean Square Error of Prediction \\
CL & Confidence Level & OLS & Ordinary Least Squares \\
GLS & Generalized Least Squares & OSTM & Ocean Surface Topography Mission \\
DW & Durbin - Watson & TG & Tide Gauge \\
ENSO & El Niño-Southern Oscillation & TOPEX & Topography Experiment \\
GMSL & Global Mean Sea Level & SA & Satellite Altimetry \\
MEI & Multivariate ENSO Index & VIF & Variance Inflation Factor \\
\hline
\end{tabular}

